\title{
25 Research Suare \\ The Impact of Hormone Receptor Testing on Breast Cancer Treatment in Dar es Salaam, Tanzania
}

\section{Magdiel Habila ( $\nabla$ magdielhabila@arizona.edu )}

The University of Arizona Mel and Enid Zuckerman College of Public Health https://orcid.org/00000003-4614-7772

\section{Jennifer Segar}

The University of Arizona Cancer Center

\section{Khadija Msami}

Ocean Road Cancer Institute

Julius Mwaiselage

Ocean Road Cancer Institute

\section{Taylor Sullivan}

City University of New York School of Public Health

\section{Mario Trejo}

The University of Arizona Mel and Enid Zuckerman College of Public Health

\section{Elizabeth Jacobs}

The University of Arizona Cancer Center

\section{Maria Bishop}

The University of Arizona Cancer Center

\section{Amr Soliman}

City University of New York School of Public Health

\section{Research article}

Keywords: breast cancer treatment, low-and-middle-income countries, cancer epidemiology, immunohistochemistry

Posted Date: July 20th, 2021

DOl: https://doi.org/10.21203/rs.3.rs-700718/v1

License: (c) (i) This work is licensed under a Creative Commons Attribution 4.0 International License. Read Full License 


\section{Abstract}

\section{Background}

Hormone receptor (HR) testing was not available for tailoring treatment of breast cancer in Tanzania until 2015. The aim of this study was to compare effect of introducing HR testing on breast cancer treatment in Tanzania.

\section{Methods}

This retrospective study included 2 groups of breast cancer patients treated at Ocean Road Cancer Institute (ORCl) before introducing HR (2007-2009 and 2014 - 685 patients), and after HR introduction (2015-2017 -765 patients). Demographic, epidemiologic and clinical data were abstracted from the medical records of patients. Chi-Square and Wilcoxon rank sum analyses were used to compare treatment in the two periods.

\section{Results}

Stage at diagnosis did not differ significantly by ER status ( $12.4 \%$ vs. $7.3 \%$, stages I and II combined, $13.4 \%$ vs. $19.3 \%$ for stage III, and $30.7 \%$ vs $37.0 \%$ for stage IV with p-values of $0.08,0.21$, and 0.44 , respectively). The proportion of patients who received both neoadjuvant chemotherapy alone $(29.2 \%$ vs $28.1 \%)$ and adjuvant chemotherapy $(53.5 \%$ vs $48.4 \%)$ did not differ significantly by ER status, $(\mathrm{p}=0.64$ and $p=0.29$, respectively). After implementing ER testing, the proportion of patients who did not receive radiotherapy differed by ER status ( $43.6 \%$ vs $32.8 \%$, $p=0.04$ ). Furthermore, approximately $13 \%$ of ERnegative patients received endocrine therapy, compared to $60 \%$ of ER positive patients $(p<0.0001)$.

\section{Discussion}

These finding suggest that there have been significant changes in breast cancer treatment practices since the introduction of hormone receptor testing. However, further research is needed to determine how hormone receptor status can be used to optimize standardized treatment protocols for patients diagnosed with breast cancer in Tanzania and other low-income countries that are taking steps to improve their breast cancer management practices and protocols.

\section{Introduction}

Breast cancer is the leading cause of cancer death among women, accounting for $11.6 \%$ of cancer deaths worldwide. ${ }^{1}$ The incidence of breast cancer is higher in low- and middle-income countries (LMICs) but developing countries have higher mortality. ${ }^{1}$ In many LMICs, there no screening programs for breast cancer and treatment options are limited. ${ }^{2}$ As a result, women often waitto be diagnosed, resulting in latestage presentation and limited treatment options. ${ }^{6}$ In addition, treatment of late-stage breast cancer is often more expensive and less effective. ${ }^{3}$ There is very little data on breast cancer incidence, mortality, 
and survival in Sub-Saharan Africa. ${ }^{3}$ The 2018 Tanzania's report on breast cancer indicated that breast cancer represented $8.12 \%$ of new cancers in the country. ${ }^{4}$ Approximately $80 \%$ of those cases were stages III and IV. ${ }^{5}$ Additionally, 17.5 per 100,000 breast cancer patients were diagnosed in 2012, and 7.8 in 100,000 died of the disease. ${ }^{4}$

The Ocean Road Cancer Institute (ORCI) is the only public cancer treatment center that provides both chemotherapy and radiotherapy in Tanzania. In Tanzania, early stage and locally advanced breast cancer undergo surgical resections first line of treatment which is performed at Muhimbili National Hospital $(\mathrm{MNH})$ in Dar es Salaam with subsequent referral to ORCI for chemotherapy and radiotherapy.

Before 2015, the standard of care at ORCI was that breast cancer patients received tamoxifen, regardless of hormone receptor (HR) status. However, starting 2015, ORCI required that patients have HR results before starting treatment. Current trends of African American breast cancer patients indicate higher estrogen receptor (ER) negative and progesterone receptor (PR)negative tumors compared to other ethnicities. ${ }^{6}$ Though these trends have not been studied extensively in representative samples in East Africa or Tanzania, ER- and PR-negative tumors have poor prognosis due to more aggressive tumor biology and lack of targeted therapy. 7,8

The aim of this study was to describe clinical characteristics of women diagnosed with breast cancer treated at $\mathrm{ORCl}$ before and after the introduction of $\mathrm{HR}$ testing. We also sought to determine the association between HR status and treatment among the study patients.

\section{Methods}

\section{Study Design}

This retrospective study included medical records of women with breast cancer treated at $\mathrm{ORCl}$ during two time periods. The time period before the introduction of HR included 685 patients treated from 20072009 and 2014, as records of patients from 2010-2013 were not available. The period after introduction of HR included 765 patients treated from January 2015 to December 2017.

\section{Study Population}

Patients seen at $\mathrm{ORCl}$ are registered in a logbook by cancer site. We used the logbooks to identify breast cancer patients treated during the study periods. Once patients were identified, corresponding medical records were retrieved and data were abstracted. All patients included in the study had primary breast cancers.

Data from the first time period before the use of ER were from our previous study Burson et al., ${ }^{5}$ and it included 488 patients treated at ORCI from July 2007 to June $2009 .{ }^{5}$ We also supplemented the first period with another dataset of primary breast cancer patients treated at ORCI between 2014 and 2015 . 
Data from the second time period after ER status were collected from 2015-2017. The number of patients in this period was 782, however, after reviewing the records, we determined that 35 patients did not have breast cancer. Additionally, records of 137 records were not available and 50 patients had duplicates that were excluded. The final number of patients of the second period was 765 . Therefore, the final total sample for the two periods combined was 1450 .

\section{Data Collection}

Data collected for the first period by Burson et al included demographic variables (age, sex, residence, body mass index (BMI)), reproductive factors (number of children, marital status, and menopausal status), clinical factors (family history of breast cancer, duration of symptoms, laterality, recurrence, stage at presentation, mass, ulceration, pain, nipple retraction, erythema, peau d'orange), pathology, and treatments (surgery and chemotherapy). ${ }^{5}$ The second group included the same variables that comprised the first period as well as HR data and endocrine therapy.

\section{Statistical Analysis}

We sought to describe the demographic trends of patients who were treated at $\mathrm{ORCl}$ in the two time periods. For these analyses, we included data on patient characteristics including age at diagnosis, BMI, and number of children. We also collected data on risk factors such as contraceptive use, alcohol use, smoking status, HIV status, insurance status, family history of breast cancer, and residence. Wilcoxon rank sum test was used for continuous variables and chi-square tests for categorical variables.

We also reviewed treatment provided compared to the National Comprehensive Cancer Network (NCCN) Framework for Resource Stratification of Guidelines: Basic Resources and the first edition of the United Republic of Tanzania National Cancer Treatment Guidelines. ${ }^{9,10}$ We used these guidelines as a standard to which we compared study results.. Based on both guidelines, we determined that HR status was an important part in the assessment of breast cancer for proper drug prescription. We conducted Fisher's exact tests to compare ER status by stage, age at diagnosis, and treatment received since these are the most important factors for determining treatment.

ER testing began at ORCI in 2015. When we reviewed the National Comprehensive Cancer Network (NCCN) Framework for Resources Stratification for NCCN Guidelines: Basic Resources ${ }^{9}$, we found that ER testing is a necessity for characterizing breast cancer, and surgery is usually the first step in treatment of early stage breast cancer. Tanzania's National Cancer Treatment Guidelines, are in accordance with the NCCN guidelines: Basic Resources. ${ }^{10}$ The guidelines recommend that women receive a core needle biopsy and immunohistochemistry be performed on tissue. Pre-operative staging is required for all patients to describe tumor size, extent of nodal involvement, and assess for symptoms and signs of metastases prior to undergoing surgery and aid in developing an appropriate treatment strategy. These guidelines also provide indications for radiation therapy, chemotherapy, and endocrine therapy based on pathology. The guidelines highlight the importance HR testing for proper drug utilization of tamoxifen for 
pre-menopausal women and anastrozole for post-menopausal women. These are available, inexpensive therapies which have disease free survival and overall survival benefit.

We also wanted to determine if the introduction of hormone receptor testing in 2015 resulted in a significant change in treatments that patients received. We assessed this difference by categorizing our data by year into the time periods before and after HR introduction. We then compared patient symptoms at diagnosis, patient characteristics, diagnostic tests, staging, and treatment within these two categories using chi-square tests. The data were analyzed using SAS Statistical Analysis Software (version 9.4). This study was approved by the institutional review boards (IRBs) of the University of Arizona and the Ethics Committee of ORCl.

\section{Results}

Table 1 shows patient characteristics, risk factors, and place of residence before and after HR testing. After implementing HR testing, there was a statistically significant increase in BMl, contraception use, and women residing in Dar es Salaam. In addition, women had fewer children, were more likely to be HIV negative, and not have access to insurance. 
Table 1

Demographics of the study population $(n=1450)$

Before HR testing

2007-2009, 2014

$N=685$

Mean $\pm \quad N(\%)$

SD

\section{Patient Characteristics ${ }^{1}$}

Age at diagnosis (years)

Body Mass Index (BMI)

Number of children carried to term

Risk Factors (Total Counted, Sum Negative Cases) 2

Use of contraceptives ${ }^{915,338}$

Use of alcohol 1450,680

Smoking status ${ }^{1450,871}$

HIV Positive 488, 450

Insurance ${ }^{1450,248}$

Family history 719,626

\section{Place of Residence}

Resident of Dar es Salaam

Residents of Other Areas and Zanzibar
$49.3 \pm$

13.14

$26.47 \pm$

5.73

$5 \pm 12.4$

Yes (\%)

119 (17.3)

$362(52.8)$

$283(41.3)$

$21(3.0)$

659 (96.2)

$35(5.1)$

123 (17.96)

197 (25.75)

$<$

0.0001

249 (36.4)

556

(81.2)

394

(57.5)

588

(85.9)

Yes (\%)

p

458 (59.8)

0.0001

408 (53.3)

0.85

296 (38.6)

0.31

17 (2.2)

0.0001

543 (70.9)

0.0001

$58(7.5)$

0.12

449 (58.7)

$<$

0.0001

${ }^{1}$ P-value calculated using Wilcoxon Rank Sum test for continuous variables

${ }^{2} \mathrm{P}$-value calculated using Chi-Square analyses for categorical variables 
Table 2 provides patient symptoms, exam findings, and diagnostic tests performed. From 2015-2017, women were more likely to present with a mass, swelling, or nipple discharge and less patients presented with pain, warmth, erythema, shiny skin, peau d'orange, or ulceration. In addition, there was an increase in the number having cytology and histology performed on tumor tissue, with the majority of patients diagnosed with invasive ductal carcinoma. Also, there was a decrease in number of women who only had mammography as part of their staging workup. 
Table 2

Symptoms, and diagnostic tests $(n=1450)$

\begin{tabular}{|c|c|c|c|}
\hline & Before HR Testing & After HR Testing & \\
\hline & 2007-2009, 2014 & $2015-2017$ & \\
\hline & $N=685$ & $N=765$ & \\
\hline Patient Symptoms Total N, Total without symptoms & Yes (\%) & Yes (\%) & \\
\hline Laterality & & & $P^{1}$ \\
\hline Left & $324(47.2)$ & $367(47.9)$ & 0.10 \\
\hline Right & $320(46.7)$ & $346(45.2)$ & 0.31 \\
\hline Bilateral & $35(5.1)$ & $28(3.6)$ & 0.37 \\
\hline Missing & $6(0.8)$ & $24(3.1)$ & 0.001 \\
\hline \multicolumn{4}{|l|}{ Symptoms } \\
\hline Mass only 1263,106 & $540(78.8)$ & $617(80.6)$ & $<0.0001$ \\
\hline Redness only 1055,957 & $72(10.5)$ & $26(3.3)$ & $<0.0001$ \\
\hline Peau d'orange only 1138,790 & $196(28.6)$ & $152(19.8)$ & $<0.0001$ \\
\hline Ulceration only 1118,796 & $168(24.5)$ & $154(20.1)$ & 0.0001 \\
\hline Nipple Retraction only 1145,837 & $154(22.4)$ & $154(20.1)$ & 0.04 \\
\hline Mass + Redness 1450,1358 & $67(9.8)$ & $25(3.3)$ & $<0.0001$ \\
\hline Mass + Peau d'orange 1450,1150 & $170(24.8)$ & $130(16.9)$ & 0.02 \\
\hline Shiny 1053,1020 & $20(2.9)$ & $13(1.6)$ & 0.02 \\
\hline \multicolumn{4}{|l|}{ Size } \\
\hline Less than $5 \mathrm{~cm}$ & $82(11.9)$ & $81(10.5)$ & 0.006 \\
\hline More than $5 \mathrm{~cm}$ & $184(26.8)$ & $293(38.3)$ & $<0.0001$ \\
\hline Swelling 1265,456 & $242(35.3)$ & $567(74.1)$ & $<0.0001$ \\
\hline Heat/ Warmth 1042, 1011 & $22(3.2)$ & $9(1.1)$ & 0.001 \\
\hline Discharge $^{750,542}$ & $41(5.9)$ & $166(21.6)$ & 0.001 \\
\hline
\end{tabular}




\begin{tabular}{|c|c|c|c|}
\hline & Before HR Testing & After HR Testing & \\
\hline Pain 1242,714 & $287(41.8)$ & $241(31.5)$ & $<0.0001$ \\
\hline Diagnostic Tests Total N, Total without test & Yes (\%) & Yes (\%) & $\mathbf{p}$ \\
\hline Cytology Only 1450,1263 & $147(21.4)$ & $40(5.2)$ & $<0.0001$ \\
\hline Histology Only 1450, 1022 & $266(38.8)$ & $162(21.2)$ & $<0.0001$ \\
\hline Cytology + Histology 1450, 957 & $206(30.0)$ & $287(37.5)$ & 0.0028 \\
\hline Mammography Only 1020, 944 & $44(6.4)$ & $32(4.1)$ & $<0.0001$ \\
\hline Breast Ultrasound 1381,1323 & $24(3.5)$ & $34(4.4)$ & 0.28 \\
\hline Abdominal Ultrasound 1373,126 & $559(81.6)$ & $688(89.9)$ & $<0.0001$ \\
\hline Chest X-Ray 1374,385 & $544(79.4)$ & $445(58.1)$ & $<0.0001$ \\
\hline Bone Scan 1389, 1204 & $79(11.5)$ & $106(13.8)$ & 0.08 \\
\hline Mammography + Breast Ultrasound 1450,1437 & $7(1.0)$ & $6(0.78)$ & 0.78 \\
\hline \multicolumn{4}{|l|}{ Histological Types Total N, Total without type } \\
\hline Carcinoma 1450,1384 & $46(6.7)$ & $20(2.6)$ & 0.0002 \\
\hline Invasive Ductal Carcinoma 1450,890 & $213(31.1)$ & $347(45.4)$ & $<0.0001$ \\
\hline Metastatic Carcinoma ${ }^{1450,1385}$ & $49(7.2)$ & $16(2.1)$ & $<0.0001$ \\
\hline Medullary Carcinoma 1450,1423 & $10(1.4)$ & $17(2.2)$ & 0.28 \\
\hline Other 1350,751 & $334(48.8)$ & $265(34.6)$ & 0.69 \\
\hline
\end{tabular}

Table 3 presents endocrine therapy use before and after HR testing was utilized. Approximately $60 \%$ of women with ER positive breast cancer received tamoxifen, while $12 \%$ had ER negative breast cancer and were treated with tamoxifen as well. Due to sparse cell counts, ER status testing frequencies were not displayed by year. However, it is important to note that in 2015, the first year ER testing began, $46 \%$ of patients who were ER positive received tamoxifen. In $2016,64 \%$ of patients who were ER-positive received tamoxifen. Finally, in $2017,87 \%$ of patients who were ER positive received tamoxifen. Data for aromatase inhibitors were not available. There was also a significant proportion of missing ER status results in these years, at $58 \%, 48 \%$, and $40 \%$, respectively. 
Table 3

Endocrine therapy stratified by ER status $(n=1450)$

\begin{tabular}{|c|c|c|c|c|}
\hline & Before HR Testing & After HR Tes & & \\
\hline & 2007-2009, 2014 & $2015-2017$ & & \\
\hline & $N=685$ & $N=765$ & & \\
\hline Therapy Type ${ }^{N}$ missing, \% missing & ER Unknown & ER Positive & ER Negative & $\mathrm{p}$ \\
\hline & $\mathrm{N}(\%)$ & $N=202$ & $N=192$ & \\
\hline & & $\mathrm{N}(\%)$ & $N(\%)$ & \\
\hline No Hormone Therapy $302,82 \%$ & $254(37.08)$ & $75(37.13)$ & $162(84.37)$ & $<0.0001$ \\
\hline Tamoxifen $43,12 \%$ & $261(38.10)$ & $120(59.41)$ & $23(11.97)$ & $<0.0001$ \\
\hline Herceptin $6,2 \%$ & $3(0.43)$ & $2(0.99)$ & $2(1.04)$ & 1.00 \\
\hline Missing 20,5\% & $167(24.37)$ & $5(2.47)$ & $5(2.60)$ & 1.00 \\
\hline
\end{tabular}

The number of ER positive and negative patients did not significantly differ by staging. See Table 4. However, prior to ER testing, the majority of patients were not staged. After starting ER testing, there was an increase in the number of patients staged. We conducted similar analyses comparing number of ER positive and negative patients by age at diagnosis (Table 5). The proportions were not significantly different by age group. 
Table 4

Stage at diagnosis stratified by ER status $(N=1450)$

\begin{tabular}{|c|c|c|c|c|}
\hline & Before HR Testing & After HR Tes & & \\
\hline & 2007-2009, 2014 & $2015-2017$ & & $P^{1}$ \\
\hline & $N=685$ & $N=765$ & & \\
\hline Stage ${ }^{N}$ missing, \% missing & ER Unknown & ER Positive & ER Negative & \\
\hline & $\mathrm{N}(\%)$ & $N=202$ & $N=192$ & \\
\hline & & $\mathrm{N}(\%)$ & $\mathrm{N}(\%)$ & \\
\hline Unknown Stage $201,54 \%$ & $668(97.52)$ & $88(43.56)$ & $70(36.46)$ & 0.15 \\
\hline Stage I - II ${ }^{15,4 \%}$ & $6(0.88)$ & 25 (12.38) & $14(7.29)$ & 0.08 \\
\hline Stage III $35,9 \%$ & $7(1.02)$ & $27(13.37)$ & $37(19.27)$ & 0.21 \\
\hline Stage IV $120,32 \%$ & $4(0.58)$ & $62(30.69)$ & 71 (36.98) & 0.44 \\
\hline
\end{tabular}


Table 5

Age distribution stratified by ER status $(n=1450)$

\begin{tabular}{|c|c|c|c|c|}
\hline & Before HR Testing & After HR Test & & \\
\hline & 2007-2009, 2014 & 2015-2017 & & $P^{1}$ \\
\hline & $N=685$ & $N=765$ & & \\
\hline $\mathrm{Age}^{\mathrm{N} \text { Missing, \% missing }}$ & ER Unknown & ER Positive & ER Negative & \\
\hline & $\mathrm{N}(\%)$ & $N=202$ & $N=192$ & \\
\hline & & $\mathrm{N}(\%)$ & $\mathrm{N}(\%)$ & \\
\hline Missing Age $68,9 \%$ & $129(18.83)$ & $26(12.87)$ & $28(14.58)$ & 0.79 \\
\hline$<19-2911,3 \%$ & $16(2.33)$ & $3(1.49)$ & $3(1.56)$ & 1.00 \\
\hline $30-3955,15 \%$ & $129(18.83)$ & $29(14.36)$ & $28(14.58)$ & 0.89 \\
\hline $40-4996,26 \%$ & $137(20.00)$ & $63(31.19)$ & $46(23.96)$ & 0.10 \\
\hline $50-5970,20 \%$ & $149(21.75)$ & $49(24.26)$ & $45(23.44)$ & 0.67 \\
\hline $60-6940,47 \%$ & $72(10.51)$ & $20(9.90)$ & $26(13.54)$ & 0.38 \\
\hline 70 and older $31,8 \%$ & $53(7.74)$ & $12(5.94)$ & $16(8.33)$ & 0.45 \\
\hline
\end{tabular}

Table 6 displays chemotherapy and radiation treatments before and after HR testing. There was a significant proportion of ER positive patients who did not receive radiation. However, $72 \%$ of patients who did not undergo radiation therapy were missing HR status data. In regards to chemotherapy, prior to ER testing, $75 \%$ of patients received neoadjuvant chemotherapy, which decreased to $15 \%$ after ER testing was implemented. There was a shift towards adjuvant chemotherapy. There was no difference in chemotherapy based on ER status, but $37 \%$ of patients, who received chemotherapy were missing HR status. 
Table 6

Treatment interventions stratified by ER status $(n=1450)$

\section{Before HR Testing \\ After HR Testing}

$\begin{array}{lll}2007-2009,2014 & 2015-2017 & P^{1} \\ N=685 & N=765 & \end{array}$

ER Unknown

ER Positive

ER Negative

$N(\%)$

$\mathrm{N}=202 \quad \mathrm{~N}=192$

$N(\%)$

$\mathrm{N}(\%)$

Chemotherapy N Missing, \% missing

\begin{tabular}{|lllll|}
\hline Neoadjuvant only $132,36 \%$ & $516(75.33)$ & $59(29.21)$ & $54(28.13)$ & 0.64 \\
\hline Adjuvant only $150,40 \%$ & $13(1.89)$ & $108(53.47)$ & $93(48.44)$ & 0.29 \\
\hline Palliative only $8,2 \%$ & $0(0)$ & $6(2.97)$ & $6(3.13)$ & 1.00 \\
\hline Neoadjuvant + Adjuvant ${ }^{10,2 \%}$ & $45(6.57)$ & $6(2.97)$ & $4(2.08)$ & 0.53 \\
\hline No Chemotherapy $35,9 \%$ & $36(5.26)$ & $11(5.45)$ & $18(9.38)$ & 0.19 \\
\hline Missing Chemotherapy ${ }^{36,10 \%}$ & $75(10.95)$ & $12(5.94)$ & $17(8.85)$ & 0.35 \\
\hline Radiotherapy N Missing, \% missing & & & & \\
\hline Yes ${ }^{86,23 \%}$ & $192(28.03)$ & $112(55.45)$ & $126(65.63)$ & 0.36 \\
\hline No ${ }^{268,72 \%}$ & $263(38.39)$ & $88(43.56)$ & $63(32.81)$ & 0.04 \\
\hline Missing ${ }^{17,5 \%}$ & $230(33.58)$ & $2(0.99)$ & $3(1.56)$ & 0.65
\end{tabular}

${ }^{1}$ P-values calculated using Chi-Square analyses, comparing proportions of ER + and ER- breast cancer patients between 2015 and 2017.

\section{Discussion}

The study revealed three main interesting observations. First, we observed that significantly more patients were treated appropriately with an increase in tamoxifen use in ER positive breast cancers and decrease use in ER negative breast cancer. There were no statistically significant differences in stage at presentation or age by ER status. In regards to treatment, there was no statistical difference in chemotherapy by ER status. However, statistically, there were more patients with ER positive breast cancer who did not receive radiation. Second, the study showed statistically significant changes in demographic factors of breast cancer patients, including increase in BMI, use of contraceptives, and 
residence in Dar es Salaam. We also found a decline in number of children and women living with HIV. Third, most common symptoms reported at presentation were breast mass, ulceration, and nipple retraction and very few patients had screening mammography before diagnosis.

Regarding the first finding related to changes in treatment after the introduction of HR testing, it is important to note that the policy of ORCI was to give tamoxifen to all breast cancer patients. However, our data indicate that less than $50 \%$ of the patients treated prior to HR testing received tamoxifen. Also, onethird of all patients received no endocrine therapy at all. One reason for this discrepancy is possibly that there was no standard treatment at this time, so physicians prescribed endocrine therapy based on their training, personal opinion, and experience. Another possibility is that the patients were seen at advanced disease stages and due to lack of standardized guidelines, they did not receive appropriate treatment. After HR testing was introduced, more than half of the patients with ER positive breast cancer received tamoxifen, however there were still a large number of patients with ER positive breast cancer who did not receive endocrine therapy (40\%). Though most patients with ER negative breast cancer did not received hormone therapy, a very small proportion ( $12 \%)$, received tamoxifen. These patients would not have yielded benefit from tamoxifen and may have been exposed to unnecessary side effects.

There was no difference in stage at diagnosis by ER status. In addition to ER status, staging is another important factor for treatment. Based on the guidelines, this includes history and physical exam, diagnostic bilateral mammogram and ultrasound if needed, and determination of HR status. Though staging is recommended to be documented before patients undergo surgical resection, our study found that before ER testing only approximately $2 \%$ of patients were staged, which improved to $53 \%$ after ER testing was introduced. While the introduction of HR testing shows a significant increase in the proportion of patients who were staged at the start of treatment, these results still show that a significant number of patients not staged when they began treatment. Another possibility is the stage was not recorded in the medical records. Our findings are consistent with other LMICs studies that show a need for standardizing staging based on the NCCN and national guidelines for Tanzania. ${ }^{9-12}$

ER status did not affect staging and $87 \%$ of patients who had stage information were stages III and IV. This raises a concern about LMICs' breast cancer patients, since the majority of them are diagnosed at advanced stages. ${ }^{13} \mathrm{~A}$ study from Nigeria found that on average, women waited 12.2 months after the onset of symptoms to seek treatment, and by the time these women were diagnosed, they were at an advanced stage. ${ }^{14}$ The delay in seeking care may be due to limited ability to recognize symptoms, not believing that the symptoms are important, fear of dying from cancer, inability to access treatment, and other sociodemographic factors including age, level of education, and seeking alternative or herbal medicne. ${ }^{3}$

There was a marginally significant increase in the proportion of patients with ER negative breast cancer who received radiation therapy. Tanzania's national guidelines advocate that radiotherapy is indicated in patients who receive lumpectomy or mastectomy with lymph node-positive disease. However, due to patient referrals from other hospitals across Tanzania and lack of accessibility of records of patients 
from remote areas, we were unable to collect the data necessary to determine which clinical factors influenced decision making for radiation therapy.

Finally, chemotherapy treatments did not differ by ER status and $83 \%$ of patients treated between 2015 and 2017 received chemotherapy. A large proportion of patients received chemotherapy regardless of ER status, possibly due to advanced stage, inherently high proportion of ER-negative tumors in Africa, and/or lack of access to gene expression profiles in predicting response to chemotherapy. Notably, in the time period before ER testing, most patients received neoadjuvant chemotherapy, however, in the time period after using HR testing, most of the patients received adjuvant chemotherapy. This could be due to the fact that chemotherapy can be used to downstage tumors before surgery. This could also represent a shift in practice or an increase in appropriate staging. However, we are unsure of what these findings suggest. Following national treatment guidelines would provide support to Tanzanian oncologists about when chemotherapy and radiotherapy would be beneficial for their patients and ensure that patients receive the best care available.

Regarding the changes in demographics, our study highlights the increase BMI and contraceptive use and decrease in pregnancies over the study period. The changes could be due the development and epidemiologic transition in low-income countries, including Tanzania. ${ }^{15}$ One of the many barriers to accessing breast cancer treatment is distance from the treatment facility. Our results indicate that there was an increase in the proportion of patients who were residents of Dar es Salaam at the time of treatment. Before HR introduction, a larger proportion of patients travelled to $\mathrm{ORCl}$ to receive treatment for breast cancer, and since then, there has been an increase in breast cancer treatment services provided in the northern parts of the country, decreasing the necessity to travel to ORCl. This finding could also be due to an increase in the population of Dar es Salaam. Patients who seek treatment must overcome transportation, financial, and socioeconomic barriers to receive treatment. ${ }^{13}$

Our results also highlighted that the majority of patients presented with large breast masses, indicating advanced disease and limited screening mammography in Tanzania. This emphasizes the need for further education of signs and symptoms as well as screening services offered to the public at ORCI in order to detect breast cancer at earlier stages.

The strengths of the study are it is the first to evaluate the impact of HR testing on treatment in Tanzania, provides an accurate description of the clinical distribution and treatment over 10 years, and was conducted at the major cancer center in Tanzania.

The limitation of the study is missing data. Until 2018, all medical records at ORCI were hand-written records. As a result, the contents of the record varied widely by physician and by information that patients shared with the medical staff. The high proportion of missing data in HR status is the primary reason our statistical tests were unable to detect differences in stage at diagnosis, age at diagnosis, and treatment based on ER status. Additionally, we were unable to collect data on the prescription of aromatase 
inhibitors and menopausal status for the patients treated at ORCl, which limits the scope of the analysis that could be conducted.

The introduction of HR testing to help determine breast cancer treatment in Tanzania is changing the paradigm of the disease. While the study results were impacted by inconsistencies in the availability of data, this study highlights the importance of implementing validated guidelines for breast cancer treatment which can serve to standardize treatment practices across physicians and decrease disparities in patient outcomes. Further research is needed to determine ORCl's compliance to breast cancer treatment guidelines, specifically relating to HR testing practices, prescribing appropriate endocrine therapy, utilizing electronic medical records to improve documentation, evaluating barriers to ER testing, and assessing the impact it has on patient outcomes.

Overall, the data supports the need to better implement ER testing and provide ER-positive patients with appropriate targeted endocrine therapy. This highlights the need for improvement in educating medical practitioners about aligning their treatment practices with national guidelines. Furthermore, in an effort to address the issues of missing information from handwritten medical records, $\mathrm{ORCl}$ recently transitioned to an electronic medical records system that is designed to assist medical staff in managing and documenting the various required aspects of treatment. ${ }^{16} \mathrm{An}$ area of further research could be to determine how effective the electronic system is at capturing patient data and treatment information. Long-term follow-up of patients with breast cancer was out of the scope for this study, however, there are guidelines now in place for addressing duration of endocrine therapy and surveillance.

\section{Conclusions}

This study found that there have been significant changes in breast cancer treatment in Tanzania over the last decade. These changes may be due to the introduction of hormone receptor status testing in 2015 , however, these changes also reflect shifts in physician practices that were observed in the changes in tamoxifen prescription among women who were ER-positive. The results of this study also highlight significant shifts in important risk factors for breast cancer including increasing BMI, decreased parity, and increased oral contraceptive use. Future research will be needed in order to determine how these changes in population characteristics might influence breast cancer diagnoses over time. These findings demonstrate that further research into the current state of risk factors associated with and management of breast cancer in low-resource settings like Tanzania is needed in order to address the disproportionate cancer burdens these populations carry.

\section{Abbreviations}

HR

hormone receptor

ER

estrogen reception 
$\mathrm{ORCl}$

Ocean Road Cancer Institute

\section{Declarations}

\section{Acknowledgements}

We would like to thank Suleiman, the research assistant who was essential in the data abstraction process, and ORCI staff for their support of this project.

Conflict of Interest Statement. The authors have no conflicts of interest.

Funding. This work was supported by Cancer Epidemiology Education in Special Populations (CEESP) Program (Grant R25 CA112383- PI, Amr Soliman)

Ethical Approval. The work was approved by the Institutional Review Board of the University of Arizona and by the Ethical Review Board of Ocean Road Cancer Institute before research was conducted.

Consent for Publication. Not applicable.

Availability of Data and Materials. All data analyzed in this project are the property of Ocean Road Cancer Institute $(\mathrm{ORCl})$ and cannot be reviewed without approval from the Ethics Committee at $\mathrm{ORCl}$.

Author Contributions. $\mathrm{MH}, \mathrm{KM}, \mathrm{JM}$, and AS developed the research project. $\mathrm{MH}, \mathrm{TS}, \mathrm{MT}$, EJ, and AS contributed to the collection, analysis, and interpretation of the data. All authors contributed to the writing of this manuscript.

\section{References}

1. The Global Cancer Observatory. Breast. 2018;876:99-100. http://gco.iarc.fr/today.

2. Kanavos P. The rising burden of cancer in the developing world. Ann Oncol. 2006;17(SUPPL. 8):1523. doi:10.1093/annonc/mdl983.

3. Harford JB. The Three Most Prominent Features of Breast Cancer in Africa: Late Diagnosis, Late Diagnosis, and Late Diagnosis. 2014:145-166.

4. The Global Cancer Observatory. Tanzania, United Republic Of:; 2018.

5. Burson AM, Soliman AS, Ngoma TA, et al. Clinical and epidemiologic profile of breast cancer in Tanzania. Breast Dis. 2010;31(1):33-41. doi:10.3233/BD-2009-0296.

6. Troester MA, Sun X, Allott EH, et al. Racial Differences in PAM50 Subtypes in the Carolina Breast Cancer Study. J Natl Cancer Inst. 2017;110(2):176-82. doi:10.1093/jnci/djx135. 
7. Mwakigonja AR, Lushina NE, Mwanga A. Characterization of hormonal receptors and human epidermal growth factor receptor-2 in tissues of women with breast cancer at Muhimbili National Hospital, Dar es Salaam, Tanzania. Infect Agent Cancer. 2017;12(1):1-12. doi:10.1186/s13027-0170170-5.

8. Rampaul RS, Pinder SE, Elston CW, Ellis IO. Prognostic and predictive factors in primary breast cancer and their role in patient management: The Nottingham breast team. Eur J Surg Oncol. 2001;27(3):229-38. doi:10.1053/ejso.2001.1114.

9. Menen RS, Teshome M. Invasive breast cancer. MD Anderson Surg Oncol Handbook Sixth Ed. 2018:31-76. doi:10.6004/jnccn.2011.0016.

10. Chaula ZAS, Kambi MB. The United Republic of Tanzania National Cancer Treatment Guidelines. Minist Heal Community Dev Gender Elder Child. 2020:1-224. doi:10.1017/CB09781107415324.004.

11. Brand NR, Qu LG, Chao A, Ilbawi AM. Delays and Barriers to Cancer Care in Low- and Middle-Income Countries: A Systematic Review. Oncologist. 2019;24(12). doi:10.1634/theoncologist.2019-0057.

12. Thomas AS, Kidwell KM, Oppong JK, et al. Breast Cancer in Ghana: Demonstrating the Need for Population-Based Cancer Registries in Low- and Middle-Income Countries. J Glob Oncol. 2017;3(6):765-72. doi:10.1200/JG0.2016.006098.

13. Yip $\mathrm{CH}$, Cazap E, Anderson BO, et al. Breast cancer management in middle-resource countries (MRCs): Consensus statement from the Breast Health Global Initiative. Breast. 2011;20(SUPPL. 2):12-9. doi:10.1016/j.breast.2011.02.015.

14. Ibrahim NA, Oludara MA. Socio-demographic factors and reasons associated with delay in breast cancer presentation: A study in Nigerian women. Breast. 2012;21(3):416-8. doi:10.1016/j.breast.2012.02.006.

15. Ford ND, Patel SA, Narayan KMV. Obesity in Low- and Middle-Income Countries: Burden, Drivers, and Emerging Challenges. Annu Rev Public Health. 2017;38:145-64.

16. Okoroafor U, Mwaiselage J, Calixte R, et al. Evaluating a newly-developed system for electronic medical records in Tanzania. An Example of the experience in low-income countries. $J$ Registry Manag. 2019;46(3):84-90. 